\title{
Electronic transport in ferromagnetic alloys and the Slater-Pauling curve
}

\author{
S. Lowitzer, ${ }^{1}$ D. Ködderitzsch, ${ }^{1}$ H. Ebert, ${ }^{1}$ and J. B. Staunton ${ }^{2}$ \\ ${ }^{1}$ Department Chemie und Biochemie, Physikalische Chemie, Universität München, Butenandstr. 5-13, 81377 München, Germany \\ ${ }^{2}$ Department of Physics, University of Warwick, Coventry CV4 7AL, United Kingdom
}

(Received 23 October 2008; published 13 March 2009)

\begin{abstract}
Experimental measurements of the residual resistivity $\rho(x)$ of the binary-alloy system $\mathrm{Fe}_{1-x} \mathrm{Cr}_{x}$ have shown anomalous concentration dependence which deviates significantly from Nordheim's rule. In the low $(x$ $<10 \%$ ) Cr concentration regime the resistivity has been found to increase linearly with $x$ until $\approx 10 \% \mathrm{Cr}$ where the resistivity reaches a plateau persisting to $\approx 20 \% \mathrm{Cr}$. In this paper we present ab initio calculations of $\rho(x)$ which explain this anomalous behavior and which are based on the Korringa-Kohn-Rostoker method in conjunction with the Kubo-Greenwood formalism. Furthermore we are able to show that the effects of short-range ordering or clustering have little effect via our use of the nonlocal coherent-potential approximation. For the interpretation of the results we study the alloy electronic structure by calculating the Bloch spectral function particularly in the vicinity of the Fermi energy. From the analysis of our results we infer that a similar behavior of the resistivity should also be obtained for iron-rich $\mathrm{Fe}_{1-x} \mathrm{~V}_{x}$ alloys - an inference confirmed by further explicit resistivity calculations. Both of these alloy systems belong to the same branch of the famous SlaterPauling plot, and we postulate that other alloy systems from this branch should show a similar behavior. Our calculations show that the appearance of the plateau in the resistivity can be attributed to the dominant contribution of minority-spin electrons to the conductivity which is nearly unaffected by the increase in $\mathrm{Cr} / \mathrm{V}$ concentration $x$, and we remark that this minority-spin electron feature is also responsible for the simple linear variation in the average moment in the Slater-Pauling plot for these materials.
\end{abstract}

DOI: 10.1103/PhysRevB.79.115109

PACS number(s): 72.15. $-\mathrm{v}, 71.20 . \mathrm{Be}$

\section{INTRODUCTION}

The Slater-Pauling plot of average magnetization per atom $M$ versus valence electron number $N_{v}$ plays a pivotal role in the understanding of the properties of ferromagnetic alloys. ${ }^{1}$ Its triangular structure of two straight lines with gradients of opposite sign neatly categorizes most alloys into one of two classes where $\frac{d M}{d N_{v}}= \pm 1$. Long ago Mott ${ }^{2}$ pointed out how this behavior can be explained by requiring the number of either majority- or minority-spin electrons to be fixed. This notion has subsequently been confirmed and given substance by modern spin density-functional theory (DFT) calculations. ${ }^{3-6}$ In this paper we follow the implications of this for the residual resistivities of ferromagnetic alloys. In particular, using the latest $a b$ initio techniques for describing disordered systems, we demonstrate how the measured apparently anomalous resistivities of iron-rich $\mathrm{Fe}_{1-x} \mathrm{Cr}_{x}$ alloys ${ }^{7,8}$ are directly attributable to their average minorityspin electron number being fixed and consequently their location on the $\frac{d M}{d N_{v}}=+1$ section of the Slater-Pauling plot. We postulate that other alloys in this category should also show the same behavior, and we strengthen this conjecture by the findings from another detailed ab initio study of $\mathrm{Fe}_{1-x} \mathrm{~V}_{x}$ alloys. We also infer that short-range order (SRO) should have little effect on the resistivities of alloys in this category, and again we are able to back up these remarks by detailed specific $a b$ initio calculations.

Many DFT calculations for disordered ferromagnetic alloys show that the majority-spin electrons "see" little disorder and that the majority-spin $d$ states are fully occupied. This leads to $\frac{d M}{d N_{v}}=-1 .^{3}$ In contrast the minority-spin electron states are significantly affected by disorder. The overall electronic transport is therefore taken up by the $s p$ majority-spin electrons. Alloys in this category include fcc-based CoMn-, FePt-, and Ni-rich NiFe alloys.
On the other hand, for some other alloys, typically Fe-rich bcc-based alloys and many Heusler alloys, the number of minority-spin $d$ electrons is fixed as the Fermi energy $E_{F}$ is pinned at a low level in a trough of the $d$-electron density of states (DOS). $\frac{d M}{d N_{v}}=+1$ of the Slater-Pauling curve follows directly from this. ${ }^{3}$ It is the ramifications of this feature for the electronic transport in such alloys that we investigate here. This time disorder is "seen" strongly by the majorityspin electrons and rather weakly by the minority-spin electrons. Figure 1 provides a relevant illustration for a bcc $\mathrm{Fe}_{0.8} \mathrm{Cr}_{0.2}$ disordered alloy. ${ }^{4}$ The Fe- and Cr-related minorityspin densities of states have similar structure in contrast to

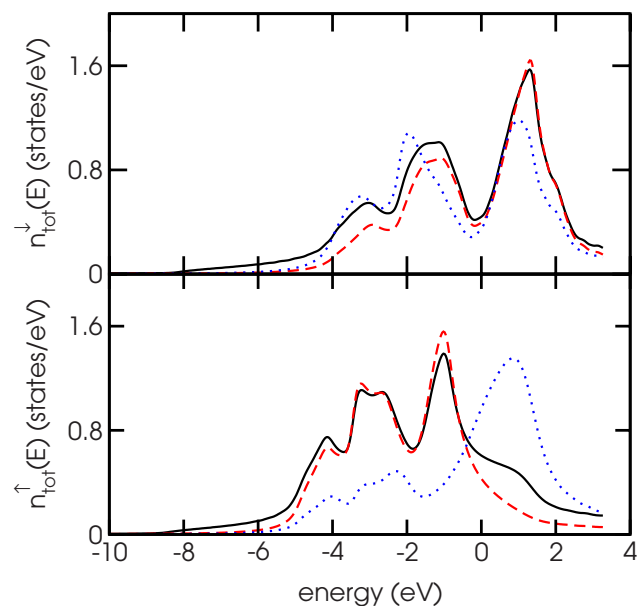

FIG. 1. (Color online) Spin-projected coherent-potential approximation (CPA) density of states of disordered bcc $\mathrm{Fe}_{0.8} \mathrm{Cr}_{0.2}$. The solid (black) line shows the total DOS, the dashed (red) line shows the Fe $d$ states and the dotted (blue) line shows the $\mathrm{Cr} d$ states. The Fermi level is located at the zero of the energy axis. 
those of majority spin. $E_{F}$ is positioned in a valley, making the average number of minority-spin electrons $\approx 3$. Moreover from these observations we can expect the resistivity to be dominated by minority-spin electrons and to be rather insensitive to overall composition and short-range order. Recently, measurements of the residual electrical resistivity of ironrich $\mathrm{Fe}_{1-x} \mathrm{Cr}_{x}$ alloys have been reported and described as anomalous. The measurements show that the resistivity increases as small amounts of $\mathrm{Cr}$ are added to $\mathrm{Fe}$ until a plateau is reached ranging from $x=10 \%$ to $20 \% .^{8}$ This behavior differs markedly from the Nordheim parabolic concentration dependence. An analysis of the data is hindered by a complexity of short-range order in $\mathrm{Fe}_{1-x} \mathrm{Cr}_{x}$. Mirebeau et al. ${ }^{9}$ reported that for $x<10 \%$ the system develops short-ranged order whereas for larger $x$ short-ranged clustering is found. At higher $\mathrm{Cr}$ concentrations still $(>20 \%)$, the alloys can undergo aging - a separation into Fe-rich $(\alpha)$ and Cr-rich $\left(\alpha^{\prime}\right)$ phases ${ }^{10,11}$ leading either to a miscibility gap or to a transformation into a tetragonal $\sigma$ phase.

We use the Kubo-Greenwood formalism ${ }^{12,13}$ implemented with the Korringa-Kohn-Rostoker (KKR) method for $a b$ initio calculations of the residual resistivities of $\mathrm{Fe}_{1-x} \mathrm{Cr}_{x}$ alloys. Disorder is accounted for by the use of the CPA. ${ }^{14}$ In order to examine the effects of short-ranged order within the disorder, we use our recently developed method which involves the nonlocal (NL)-CPA. ${ }^{15}$ We complement our $a b$ initio resistivity calculations with Bloch spectral-function (BSF) calculations which show the electronic structure in the vicinity of $E_{F}$ and enable a connection to be made to the semiclassical Boltzmann description of electronic transport properties. ${ }^{16}$

We find that our calculations fully support our initial expectations outlined above which are linked to the alloy location on the Slater-Pauling plot in addition to describing the experimental data well. Moreover from further calculations for $\mathrm{Fe}_{1-x} \mathrm{~V}_{x}$ alloys we predict that a similar behavior will be found for these materials as well as for other systems sited in this region of the Slater-Pauling plot.

Section II outlines the $a b$ initio theoretical framework (full details are given in Refs. 14 and 15). This is followed by our study of the resistivity of $\mathrm{Fe}_{1-x} \mathrm{Cr}_{x}$ as a function of $x$ together with Bloch spectral-function interpretation. We then describe a similar investigation of $\mathrm{Fe}_{1-x} \mathrm{~V}_{x}$ alloys. Section IV summarizes and emphasizes the overall conclusions.

\section{THEORY}

\section{A. Kubo-Greenwood equation}

The basis of our investigations is supplied by the KuboGreenwood equation for the symmetric part of the conductivity tensor, ${ }^{14}$

$$
\sigma_{\mu \nu}=\frac{\hbar}{\pi N \Omega} \operatorname{Tr}\left\langle\hat{j}_{\mu} \Im G^{+}\left(E_{F}\right) \hat{j}_{\nu} \Im G^{+}\left(E_{F}\right)\right\rangle .
$$

Here $N$ is the number of atomic sites, $\Omega$ is the volume per atom, \langle\rangle indicates an averaging over configurations, and $\Im G^{+}\left(E_{F}\right)$ is the imaginary part of the retarded one-particle Green's function at the Fermi energy $E_{F}$. For the determination of $\Im G^{+}\left(E_{F}\right)$ we use the multiple-scattering theory (MST) which is the basis of the KKR band-structure method. Within MST the real-space representation of $\Im G^{+}$has the following form: ${ }^{17}$

$$
\Im G^{+}\left(\vec{r}, \vec{r}^{\prime}, E\right)=\mathfrak{I} \sum_{\Lambda_{1} \Lambda_{2}} Z_{\Lambda_{1}}(\vec{r}, E) \tau_{\Lambda_{1} \Lambda_{2}}(E) Z_{\Lambda_{2}}^{\times}\left(\vec{r}^{\prime}, E\right) .
$$

Using a fully relativistic implementation, the wave functions $Z\left(Z^{\times}\right)$are the regular right (left-)-hand side solutions of the Dirac equation, $\tau$ is the scattering path operator, and $\Lambda$ $=(\kappa, \mu)$ with $\kappa$ and $\mu$ being the relativistic spin orbit and magnetic quantum numbers. ${ }^{18}$ Finally, $\hat{j}_{\mu}$ in Eq. (1) is the current-density operator that is given in relativistic form by

$$
\hat{j}_{\mu}=e c \boldsymbol{\alpha}_{\mu},
$$

where $\boldsymbol{\alpha}_{\mu}$ is one of the standard Dirac matrices. ${ }^{18}$

The central step to calculate the conductivity is to handle the averaging over all possible configurations. For a randomly disordered system the CPA (Ref. 19) is a wellestablished method for the configuration averaging. The CPA introduces an effective medium which represents the electronic properties of the disordered alloy. This effective medium is described by its single-site $t$ matrix $t_{\mathrm{CPA}}$ in combination with its averaged scattering path operator $\tau_{\mathrm{CPA}}$,

$$
\underline{\tau}_{\mathrm{CPA}}=\frac{1}{\Omega_{\mathrm{BZ}}} \int_{\Omega_{\mathrm{BZ}}} d^{3} k\left[\left(\underline{t}_{\mathrm{CPA}}\right)^{-1}-\underline{G}(\vec{k}, E)\right]^{-1},
$$

where the underline indicates matrices with respect to the spin-angular character $\Lambda$. Assuming a binary-alloy $A_{1-x} B_{x}$, $\underline{t}_{\mathrm{CPA}}$ and $\underline{\tau}_{\mathrm{CPA}}$ have to fulfill the CPA condition

$$
\underline{\tau}_{\mathrm{CPA}}=(1-x) \underline{\tau}_{A}+x \underline{\tau}_{B} .
$$

$\underline{\tau}_{A(B)}$ is the scattering path operator for an atom of type $A(B)$ embedded into the CPA medium and $G(\vec{k}, E)$ is the $\mathrm{KKR}$ structure constant matrix. With these CPA equations it is possible to construct the effective medium iteratively.

Butler ${ }^{14}$ derived within the CPA a scheme to handle the averaging over configurations of the two Green's functions. His formulation of the Kubo-Greenwood equation on the basis of the KKR CPA is widely applied and gives in general good agreement with experimental data. ${ }^{20-25}$

It has to be emphasized that the CPA is a single-site theory and therefore neglects correlations concerning the occupation of the neighboring atomic sites. For that reason one has to use more elaborate methods to include SRO effects. One possibility is to use the NL-CPA. ${ }^{26-30}$ The NL-CPA creates (as the CPA) an effective medium and can be understood as a cluster generalization of the well-established CPA. The corresponding NL-CPA equations can be written as

$$
\begin{aligned}
\underline{\underline{\mathrm{N}}}_{\mathrm{NL}-\mathrm{CPA}}^{J J}= & \frac{1}{\Omega_{\mathrm{BZ}}} \sum_{\vec{K}_{n}}\left\{\int _ { \Omega _ { K _ { n } } } d ^ { 3 } k \left[\left(\underline{t}_{\mathrm{NL}-\mathrm{CPA}}\right)^{-1}-\underline{G}(\vec{k}, E)\right.\right. \\
& \left.\left.-\delta \hat{\underline{G}}\left(\vec{K}_{n}, E\right)\right]^{-1}\right\} e^{i \vec{K}_{n} \cdot\left(\vec{R}_{I}-\vec{R}_{J}\right)}, \\
\underline{\underline{\tau}}_{\mathrm{NL}-\mathrm{CPA}} & =\sum_{\gamma} P_{\gamma} \underline{\underline{\tau}}_{\gamma}\left(\text { with } \sum_{\gamma} P_{\gamma}=1\right),
\end{aligned}
$$


with the double underline indicating matrices with respect to site and spin-angular character indices $I$ and $\Lambda$, respectively. In Eq. (7) $\underline{\tau}_{\gamma}$ is the scattering path operator for a cluster of type $\gamma$ embedded into the NL-CPA medium, $P_{\gamma}$ is the probability that this cluster type occurs, and $\delta \hat{G}\left(\vec{K}_{n}, E\right)$ are effective structure constant corrections for tile $\vec{K}_{n}$ which account for nonlocal correlations due to the disorder configurations. ${ }^{27}$

Recently the KKR NL-CPA was combined with the Kubo-Greenwood equation. ${ }^{15}$ On the basis of this scheme it is possible to investigate ordering effects on the residual resistivity in a systematic way.

For ferromagnetic cubic solids with the magnetization along the $z$ axis the conductivity tensor consists of three independent components: $\sigma_{x x}=\sigma_{y y}=\sigma_{\perp}, \sigma_{z z}=\sigma_{\|}$, and $\sigma_{x y}=$ $-\sigma_{y x} . \sigma_{\perp}$ and $\sigma_{\|}$are the transverse and longitudinal conductivities, while $\sigma_{x y(y x)}$ determine the spontaneous or anomalous Hall resistivity. In line with the abovementioned experimental investigations only the isotropic resistivity $\bar{\rho}$ is considered that is given by

$$
\bar{\rho}=\frac{1}{3}\left(2 \rho_{\perp}+\rho_{\|}\right)
$$

with $\rho_{\perp}=\sigma_{\perp}^{-1}$ and $\rho_{\|}=\sigma_{\|}^{-1}$ if the spin-orbit-induced component $\sigma_{x y}$ is ignored.

\section{B. Bloch spectral functions} $\operatorname{as}^{31}$

In a most general way the density of states may be defined

$$
n(E)=\sum_{n} \delta\left(E-E_{n}\right)
$$

where $E_{n}$ are the electronic eigenvalues of the system. In analogy the BSF can be defined by ${ }^{17}$

$$
A(E, \vec{k})=\sum_{n} \delta\left[E-E_{n}(\vec{k})\right],
$$

and for that reason it can be regarded as a $\vec{k}$-resolved density of state. Dealing with an ordered system and a given $\vec{k}$ vector the BSF has, at the positions of the eigenvalues, an infinitely sharp peak and it is zero everywhere else. If one has an alloy instead of a perfect crystal an appropriate expression for the BSF within KKR CPA was worked out by Faulkner and Stocks, ${ }^{17}$

$$
A(E, \vec{k})=-\frac{1}{\pi} \mathfrak{I} \operatorname{Tr}\left[\underline{F}^{c c} \underline{\tau}_{\mathrm{CPA}}(E, \vec{k})\right]-\frac{1}{\pi} \mathfrak{I} \operatorname{Tr}\left[\left(\underline{F}^{c}-\underline{F}^{c c}\right) \underline{\tau}_{\mathrm{CPA}}\right],
$$

with

$$
\underline{\tau}_{\mathrm{CPA}}=\frac{1}{\Omega_{\mathrm{BZ}}} \int_{\mathrm{BZ}} d^{3} k \underline{\tau}_{\mathrm{CPA}}(E, \vec{k}) .
$$

The matrices $\underline{F}^{c}$ and $\underline{F}^{c c}$ are given in terms of the overlap integrals

$$
F_{\Lambda \Lambda^{\prime}}^{\alpha \beta}=\int_{\Omega} d^{3} r Z_{\Lambda}^{\alpha \times}(E, \vec{r}) Z_{\Lambda^{\prime}}^{\beta}(E, \vec{r}) .
$$

$\alpha$ and $\beta$ denotes an atom type of the alloy. For more details and explicit expressions see Ref. 17. Compared to a pure

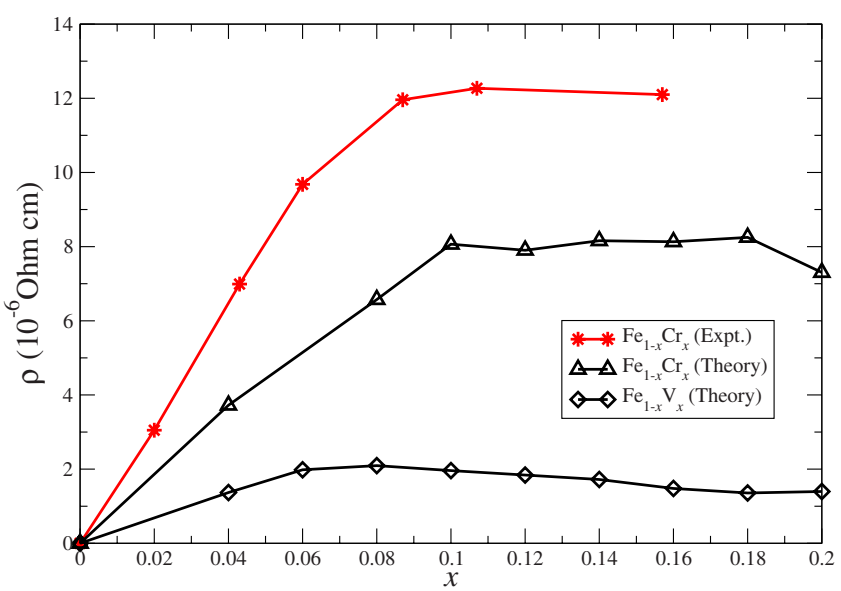

FIG. 2. (Color online) The residual resistivity of $\mathrm{Fe}_{1-x} \mathrm{Cr}_{x}$ and $\mathrm{Fe}_{1-x} \mathrm{~V}_{x}$ as a function of the $\mathrm{Cr} / \mathrm{V}$ concentration $x$. The asterisks (red line) show the experimental data for $\mathrm{Fe}_{1-x} \mathrm{Cr}_{x}$ of Ref. 7 at $4.2 \mathrm{~K}$. The triangles and diamonds (black lines) show our CPA results for $\mathrm{Fe}_{1-x} \mathrm{Cr}_{x}$ and $\mathrm{Fe}_{1-x} \mathrm{~V}_{x}$, respectively.

system, the BSF for an alloy becomes broadened due to the disorder. This broadening can be related to the lifetime of an electron in a Bloch state and is therefore quite useful for the interpretation of the resistivity data. ${ }^{16}$

With the BSF it is possible to discuss a dispersion relation $E(\vec{k})$ even for alloys. ${ }^{32}$ Strictly speaking such a dispersion relation is in general not defined for alloys because $\vec{k}$ is not a good quantum number for disordered systems. Nevertheless, the dispersion relation represented by the BSF can be used to calculate Fermi velocities ${ }^{16}$ and gives therefore useful hints for the interpretation of resistivity data.

\section{Computational details}

All calculations were done in the framework of spin density-functional theory using the local spin-density approximation (LSDA) with the parametrization of Vosko et al. ${ }^{33}$ for the exchange-correlation potential.

The CPA and the NL-CPA formulations of the KuboGreenwood equation were implemented within the selfconsistent spin-polarized relativistic KKR (SPR-KKR) scheme. ${ }^{34}$ All calculations include vertex corrections. ${ }^{14,15}$ To ensure convergence of the results with respect to the angularmomentum expansion, the cutoff $l_{\max }=4$ has been used.

\section{RESULTS}

\section{A. CPA Results}

The central result of our calculations is shown in Fig. 2. This figure shows the residual resistivity of $\mathrm{Fe}_{1-x} \mathrm{Cr}_{x}$ and $\mathrm{Fe}_{1-x} \mathrm{~V}_{x}$ as a function of the $\mathrm{Cr} / \mathrm{V}$ concentration $x$. As mentioned above, the experimental $\mathrm{Fe}_{1-x} \mathrm{Cr}_{x}$ data show an anomalous behavior. In the low $\mathrm{Cr}$ concentration regime $(<\approx 10 \%)$ the residual resistivity increases with increasing $\mathrm{Cr}$ concentration. Further increase in the $\mathrm{Cr}$ content does not lead to a further increase in the resistivity. Our theoretical results show the same variation with the $\mathrm{Cr}$ concentration. At $10 \% \mathrm{Cr}$ we obtain the highest value for the resistivity. If one 

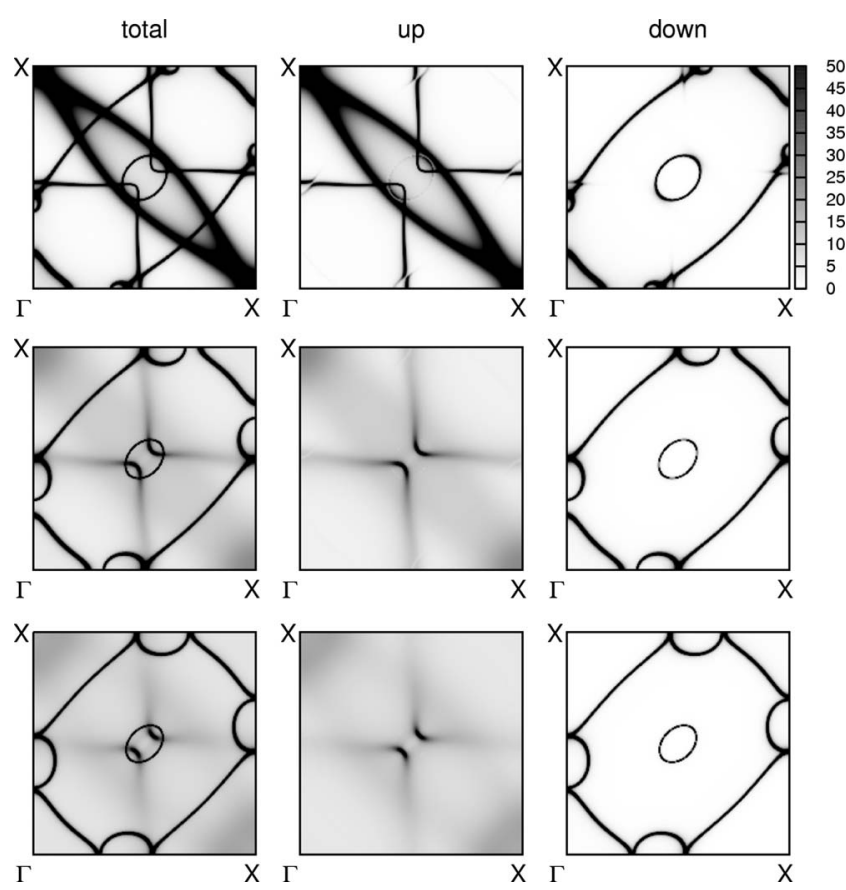

FIG. 3. Total and spin-projected BSF of $\mathrm{Fe}_{1-x} \mathrm{Cr}_{x}$ at the Fermi energy in the (001) plane for different $\mathrm{Cr}$ concentrations (top: $4 \%$ $\mathrm{Cr}$; middle: $12 \% \mathrm{Cr}$; bottom: $20 \% \mathrm{Cr}$ ). The black regions correspond to values $>50 \mathrm{arb}$. units. For a better resolution the cusps of the BSF have been cut.

further increases the $\mathrm{Cr}$ concentration, the resistivity stays more or less constant at $\approx 8 \mu \Omega \mathrm{cm}$.

The $\mathrm{Fe}_{1-x} \mathrm{~V}_{x}$ alloys show a similar behavior for the theoretical residual resistivity. With increasing $\mathrm{V}$ concentration the residual resistivity increases up to $\approx 2 \mu \Omega \mathrm{cm}$ (at $6 \% \mathrm{~V}$ ). Further increase in the $\mathrm{V}$ concentration leads only to small changes in the residual resistivity.

This behavior can be explained by a different variation in the electronic structure for the majority- and minority-spin subsystems when the $\mathrm{Cr} / \mathrm{V}$ concentration changes. Adding $\mathrm{Cr}$ to pure $\mathrm{Fe}$ in a random way, the disorder in the system increases, and with this the resistivity increases. This conventional behavior is observed in the regime with a $\mathrm{Cr}$ content smaller than $\approx 8 \% \mathrm{Cr}$ where the system shows a Nordheimtype behavior. To identify the contribution of the majority-/ minority-spin subsystems to the conductivity, we calculated the BSF according to Eq. (11). Figure 3 shows the total and spin-projected BSF for three different $\mathrm{Cr}$ concentrations (4\%, $12 \%$, and $20 \% \mathrm{Cr}$ ). The important observation from the displayed BSF is the different dependencies of the majorityand minority-spin subsystems on the $\mathrm{Cr}$ concentration. At 4\% Cr both spin subsystems show sharp peaks for the BSF which indicate that the impact of disorder is relatively small. If one increases the $\mathrm{Cr}$ concentration up to $12 \%$ a dramatic change occurs. For the BSF of the majority subsystem the prominent lens-shaped band disappears and the remaining rectangular-shaped band becomes strongly smeared out, whereas the minority component is almost unchanged. Further increase in the $\mathrm{Cr}$ concentration continues this trend. For a better illustration of the influence of the $\mathrm{Cr}$ increase in the minority component, we show in Fig. 4 explicitly the peaks

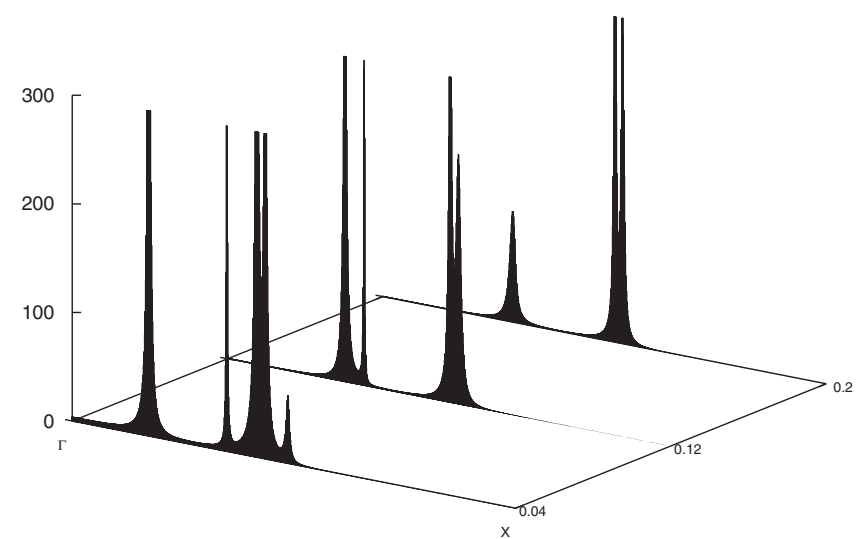

FIG. 4. BSF along the $\Gamma-X$ direction for the minority-spin subsystem in $\mathrm{Fe}_{1-x} \mathrm{Cr}_{x}$ for three different $\mathrm{Cr}$ concentrations (4\%, 12\%, and $20 \% \mathrm{Cr}$ ). The cusps of the BSF have been cut at 300 arb. units.

of the BSF at the Fermi energy along the $\Gamma-X$ direction. The BSF shows three main peaks with the last peak (the closest peak to the $X$ point) being split. At $4 \% \mathrm{Cr}$ an additional small peak is present to the right of the split peak. This peak is due to a hybridization with the majority subsystem. This can be inferred from Fig. 3 if one compares the minority and majority BSFs for $4 \% \mathrm{Cr}$. The reason for this hybridization is that in fully relativistic calculations, the spin is not a good quantum number because of the presence of spin-orbit coupling..$^{35}$

The behavior of the three remaining peaks is quite different. The narrow peak shifts with increasing $\mathrm{Cr}$ concentration toward the $\Gamma$ point, and at $20 \% \mathrm{Cr}$ this peak overlaps with the peak closest to the $\Gamma$ point. The split peak remains nearly fixed at its position but one observes a narrowing with increasing $\mathrm{Cr}$ concentration, which corresponds to an increased lifetime of this state.

For comparison we also calculated BSF for $\mathrm{Fe}_{1-x} \mathrm{~V}_{x}$ and found a similar behavior of the majority-/minority-spin subsystem as for $\mathrm{Fe}_{1-x} \mathrm{Cr}_{x}$. Figure 5 shows the spin-projected BSF for $\mathrm{Fe}_{0.8} \mathrm{~V}_{0.2}$. One can see that again the majority component becomes smeared out whereas the minority component displays sharp peaks. To get a more detailed picture of the $\mathrm{Fe}_{1-x} \mathrm{~V}_{x} \mathrm{BSF}$ we show in Fig. 6 a similar picture as shown in Fig. 4 for $\mathrm{Fe}_{1-x} \mathrm{Cr}_{x}$. If one compares Fig. 6 with Fig. 4 one can see that for $\mathrm{Fe}_{1-x} \mathrm{~V}_{x}$ the BSF peaks are sharper than for $\mathrm{Fe}_{1-x} \mathrm{Cr}_{x}$. Therefore one can say that the minorityspin electrons see a smaller difference between $\mathrm{Fe}$ and $\mathrm{V}$ atoms compared to $\mathrm{Fe}$ and $\mathrm{Cr}$ atoms. This explains why the
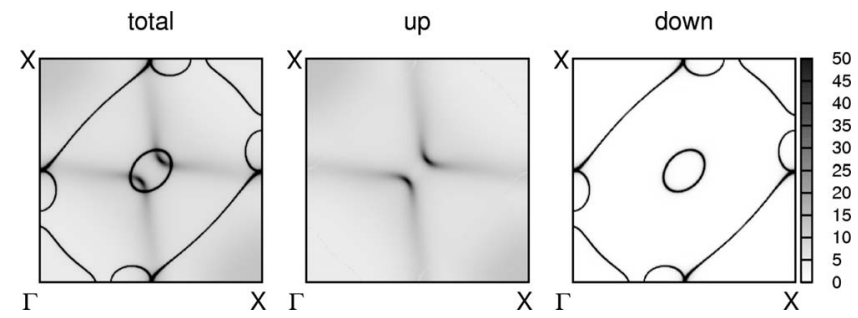

FIG. 5. Total and spin-projected BSF of $\mathrm{Fe}_{0.8} \mathrm{~V}_{0.2}$ at the Fermi energy in the (001) plane. The black regions correspond to values $>50$ arb. units. For a better resolution the cusps of the BSF have been cut. 


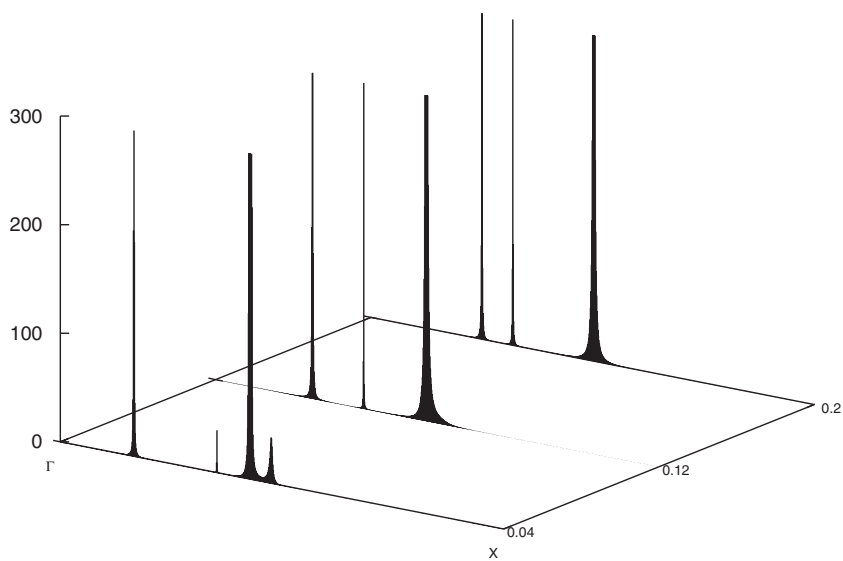

FIG. 6. BSF along the $\Gamma-X$ direction for the minority-spin subsystem in $\mathrm{Fe}_{1-x} \mathrm{~V}_{x}$ for three different $\mathrm{V}$ concentrations $(4 \%, 12 \%$, and $20 \% \mathrm{~V}$ ). The cusps of the BSF have been cut at 300 arb. units.

residual resistivities are higher in $\mathrm{Fe}_{1-x} \mathrm{Cr}_{x}$ compared to $\mathrm{Fe}_{1-x} \mathrm{~V}_{x}$. Figure 6 shows that the increased disorder due to the increased V concentration does not affect the BSF peaks of the minority-spin subsystem. Jen and Chang ${ }^{36}$ measured the residual resistivity of polycrystalline $\mathrm{Fe}_{1-x} \mathrm{~V}_{x}$. They observed a monotonically increase in the residual resistivity in the range of $0.04<x<0.2$. They also measured the anisotropic magnetoresistance and obtained a maximum for this quantity at $\approx 7 \% \mathrm{~V}$. The direct comparison of these measurements with our transport calculations is difficult, however, owing to the influence of the polycrystalline nature of the samples on the resistivity being hard to estimate.

To identify the character of the smeared out states from the majority subsystem, we projected the $\mathrm{Fe}_{1-x} \mathrm{Cr}_{x} \mathrm{BSF}$ according to its $s, p$, and $d$ contributions. This is shown in Fig. 7. The main part of the majority states has $d$-like character. These states obviously strongly broaden with increasing $\mathrm{Cr}$ concentration. This behavior is opposite to that of the minority subsystem, although this is also dominated by $d$-like states. The different behavior of the $d$ states for the two spin subsystems can also be seen in the density of states. In Figs. 1 and 8 the spin-projected DOS of $\mathrm{Fe}_{1-x} \mathrm{Cr}_{x}$ for two different $\mathrm{Cr}$ concentrations is shown. In addition to the total DOS we show the $d$-like part of the DOS. The DOS shown in Fig. 8 is very close to that of pure $\mathrm{Fe}$. One can see that for the majority component the antibonding Fe $d$ states are also occupied whereas for the minority component the Fermi level is located in a so-called pseudogap below the antibonding states. Figure 1 clearly shows the relative positions of the $\mathrm{Cr}$

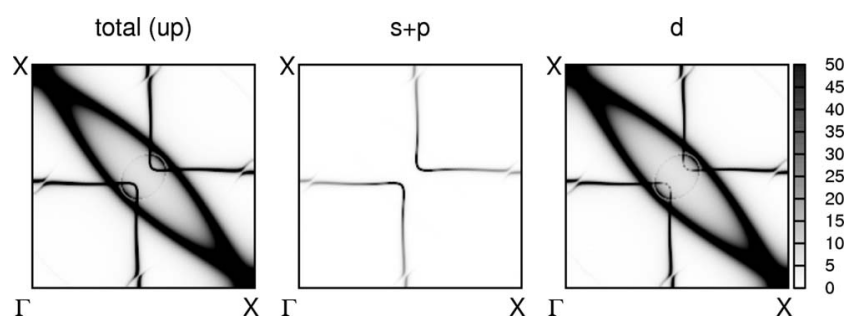

FIG. 7. Projected majority component of the $\mathrm{Fe}_{1-x} \mathrm{Cr}_{x}$ BSF at $4 \% \mathrm{Cr}$. The left plot shows the total BSF whereas the middle and the right plots show the $s+p$ - and $d$-projected BSFs, respectively.

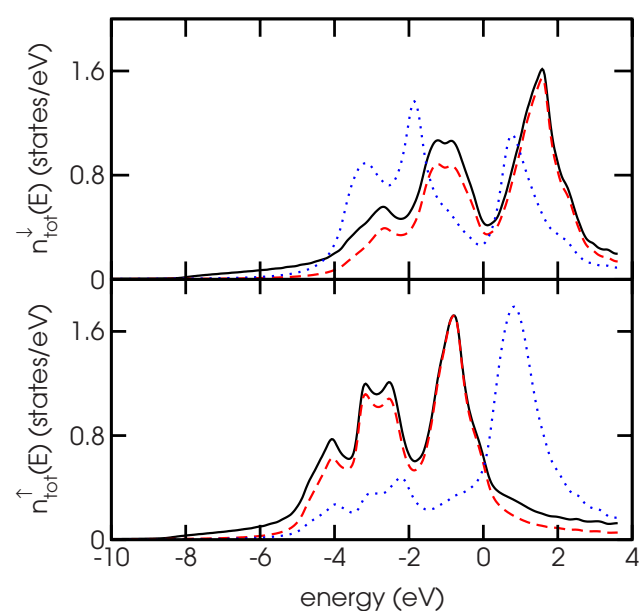

FIG. 8. (Color online) Spin-projected CPA density of states of $\mathrm{Fe}_{0.96} \mathrm{Cr}_{0.04}$. The solid (black) line shows the total DOS, the dashed (red) line shows the Fe $d$ states, and the dotted (blue) line shows the $\mathrm{Cr} d$ states. The Fermi level is located at the zero of the energy axis.

and Fe $d$ states. These states are strongly hybridized for the minority component. The opposite happens in the majorityspin channel, where the $\mathrm{Fe}$ and the $\mathrm{Cr} d$ states are well separated in energy.

With increasing $\mathrm{Cr}$ concentration the antibonding $\mathrm{Fe} d$ peak of the majority component becomes more and more depopulated and new states appear above the Fermi level. Olsson et al. ${ }^{11}$ showed that this leads to a completely smeared out band at approximately equiatomic composition. If one compares this with the behavior of the $d$ states of the minority component, one can see that the increase in $\mathrm{Cr}$ concentration has no effect on the total DOS of the minority component.

It is well known that the DOS of $\mathrm{Fe}_{1-x} \mathrm{~V}_{x}$ consists of a minority-spin subsystem where the Fermi energy is pinned in a pseudogap and a majority-spin subsystem which becomes broadened and depopulated with increasing $\mathrm{V}$ concentration. ${ }^{4,6}$ These similar characteristics of the $\mathrm{Fe}_{1-x} \mathrm{Cr}_{x}$ and the $\mathrm{Fe}_{1-x} \mathrm{~V}_{x}$ DOSs are responsible for the appearance of the Slater-Pauling curve for the magnetic moment of these alloys. Due to the fact that the number of minority electrons $\left(N_{\downarrow}\right)$ is independent of the $\mathrm{Cr} / \mathrm{V}$ concentration, the magnetization per atom $M$ varies linearly with the $\mathrm{Cr} / \mathrm{V}$ concentration, ${ }^{4}$

$$
M=Z-2 N_{d \downarrow}-2 N_{s p \downarrow},
$$

with $Z$ as the number of valence electrons. The number of $s p$ electrons in the minority-spin system $N_{s p \downarrow}$ changes only very little across the $3 d$ row. ${ }^{4}$ Therefore one can conclude that the $\mathrm{Cr} / \mathrm{V}$ concentration-independent hybridized $\mathrm{Fe}$ and $\mathrm{Cr} / \mathrm{V} d$ states of the minority-spin subsystem are responsible for the appearance of the Slater-Pauling curve and in addition for the apparently anomalous behavior of the residual resistivity of these materials.

The discussion of the electronic structure of $\mathrm{Fe}_{1-x} \mathrm{Cr}_{x}$ and $\mathrm{Fe}_{1-x} \mathrm{~V}_{x}$ in terms of the BSF and DOS curves has obviously been made in the spirit of the two-current model for the conductivity of spin-polarized solids. The fully relativistic 


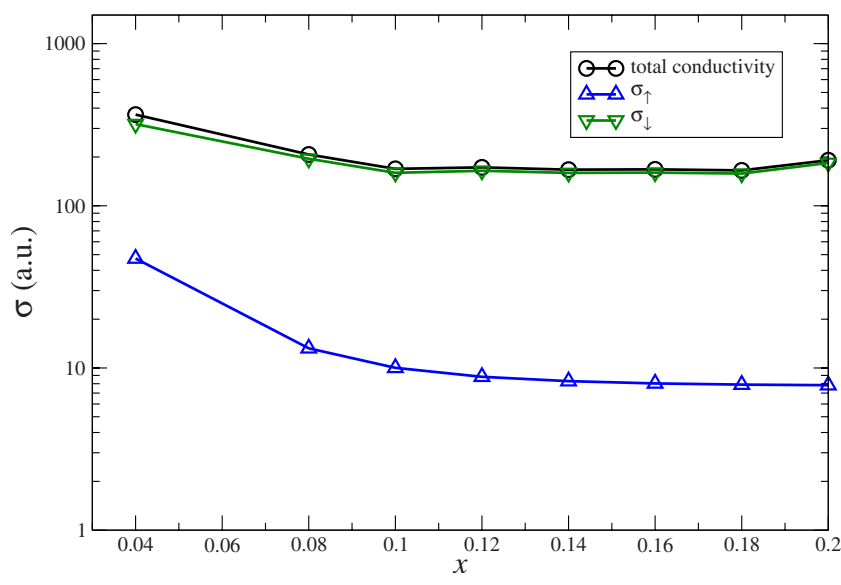

FIG. 9. (Color online) Spin-resolved conductivities $\sigma_{\uparrow}$ and $\sigma_{\downarrow}$ for $\mathrm{Fe}_{1-x} \mathrm{Cr}_{x}$.

approach for calculating the conductivity used here, strictly speaking, does not allow a decomposition of the conductivity into spin channels. The reason for this is that spin-orbit coupling gives rise not only to the off-diagonal elements $\sigma_{x y}$ mentioned above but also to spin-flip contributions that influence the isotropic conductivity or resistivity, respectively. If the spin-orbit coupling strength is not too strong, however, an approximate decomposition can nevertheless be made. As was demonstrated in Ref. 37, ignoring the small spin-flip elements connected with the current-density operator in Eq. (3), one can still define a spin-projected conductivity. This is indeed justified for $\mathrm{Fe}_{1-x} \mathrm{Cr}_{x}$ by the fact that the sum of the approximate conductivities $\sigma_{\uparrow}+\sigma_{\downarrow}$ hardly differs from the conductivity calculated in a relativistic way (Fig. 2). The results for $\sigma_{\uparrow}$ and $\sigma_{\downarrow}$ shown in Fig. 9 indeed confirm the picture that evolved from the BSF, i.e., $\sigma_{\downarrow}$ is about 2 orders of magnitude larger than $\sigma_{\uparrow}$ and is nearly concentration independent for $x>8 \%$.

In summary the resistivity increase from $0 \%$ to $10 \% \mathrm{Cr}$ $(0 \%$ to $6 \% \mathrm{~V})$ is due to the increased disorder scattering for the majority-spin subsystem; roughly speaking, the contribution of the majority subsystem to the conductivity drops down. This drop down can be explained by a smeared out BSF for this component. The increasing $\mathrm{Cr} / \mathrm{V}$ concentration leads to a broadening of the BSF. This broadening can be related to a decrease in the lifetime of the investigated electronic state which leads to an increasing resistivity. At higher $\mathrm{Cr} / \mathrm{V}$ concentrations only the minority subsystem contributes to the conductivity. The increase in the $\mathrm{Cr} / \mathrm{V}$ concentration leads to no broadening of the minority states. Therefore, the contribution of this component to the conductivity in the range of $10 \%-20 \% \mathrm{Cr}(6 \%-20 \% \mathrm{~V})$ is constant. This leads to a nearly constant resistivity in that concentration regime.

\section{B. NL-CPA results}

The next step in our analysis is to investigate the influence of SRO effects on the residual resistivity since this was suggested to be the reason for the anomalous concentration dependence of the residual resistivity. To include SRO effects we use the NL-CPA which is, as explained above, a cluster

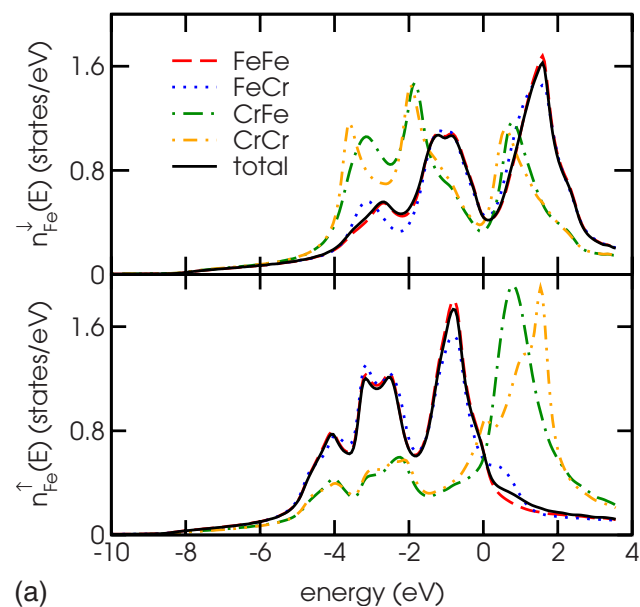

(a)

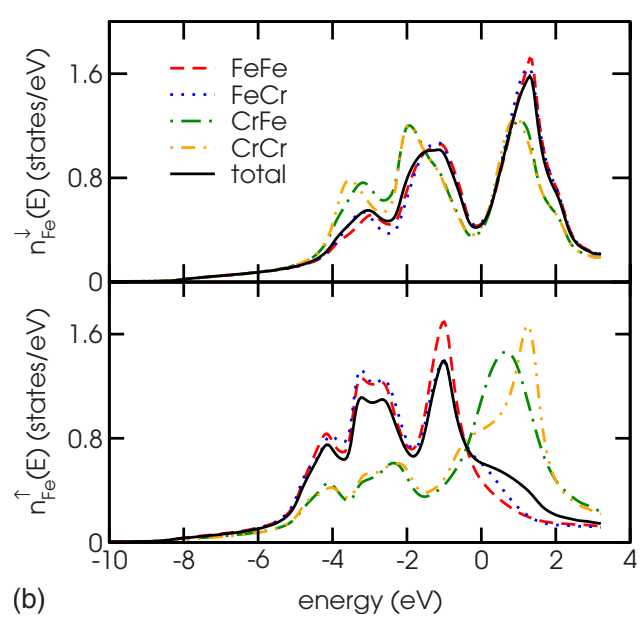

FIG. 10. (Color online) Cluster-resolved NL-CPA density of states of disordered $\mathrm{Fe}_{1-x} \mathrm{Cr}_{x}$ (top: $4 \% \mathrm{Cr}$; bottom: $20 \% \mathrm{Cr}$ ). The solid (black) line shows the total DOS, the dashed (red) line shows the contribution of the FeFe cluster, the (blue) dotted line of the $\mathrm{FeCr}$ cluster, the (green) dashed-dotted line of the $\mathrm{CrFe}$ cluster, and the (orange) dashed-dotted-dotted line of the $\mathrm{CrCr}$ cluster.

generalization of the CPA. Therefore we have to define appropriate cluster configurations and their associated probabilities $\left(P_{\gamma}\right)$. We used the smallest possible bcc cluster with two atoms. This leads to four different cluster configurations, e.g., $\mathrm{Fe}_{1-x} \mathrm{Cr}_{x}$ : pure $\mathrm{Fe}(\mathrm{FeFe})$, pure $\mathrm{Cr}(\mathrm{CrCr})$, and two mixed clusters with different occupation of the lattice sites ( $\mathrm{FeCr}$ and $\mathrm{CrFe}$ ). The probabilities of the configurations depend on the investigated ordering case. For example in the case of $\mathrm{Fe}_{0.5} \mathrm{Cr}_{0.5}$ and $\mathrm{SRO}$, only the configurations $\mathrm{FeCr}$ and $\mathrm{CrFe}$ have to be considered. For the simulation of clustering effects only the pure configurations $\mathrm{FeFe}$ and $\mathrm{CrCr}$ have nonzero probabilities, and to simulate total disorder all configurations are used. In Ref. 15 a detailed description of how to define the configuration probabilities for a bcc lattice is given. To display the contribution of the different cluster configurations to the density of states of a disordered $\mathrm{Fe}_{1-x} \mathrm{Cr}_{x}$ crystal, we show in Fig. 10 the cluster-resolved density of states for two different $\mathrm{Cr}$ concentrations. From this figure one can see that the total DOS agrees very well with the total CPA DOS from Figs. 1 and 8. The most dominant contribution to the total DOS comes from the $\mathrm{FeFe}$ cluster 


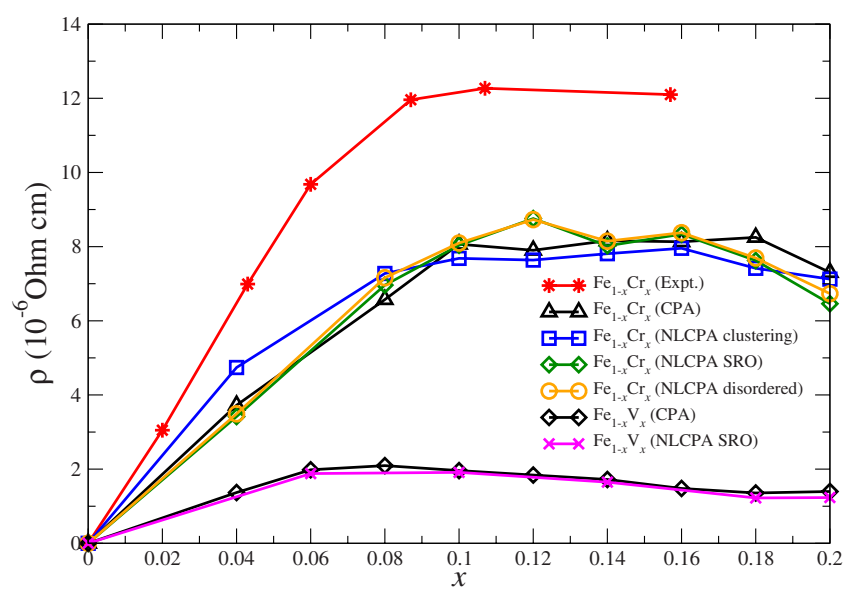

FIG. 11. (Color online) The resistivity of $\mathrm{Fe}_{1-x} \mathrm{Cr}_{x}$ and $\mathrm{Fe}_{1-x} \mathrm{~V}_{x}$ as a function of the concentration $x . \mathrm{Fe}_{1-x} \mathrm{Cr}_{x}$ : the asterisks (red line) show the experimental data of Ref. 7 and the triangles (black line) show our CPA results. The circles (yellow line) show the NLCPA results for the disordered alloys; the diamonds (green line) show results when SRO is included and the squares (blue line) when short-range clustering is included. $\mathrm{Fe}_{1-x} \mathrm{~V}_{x}$ : the diamonds (black line) show our CPA results and the crosses (magenta line) show results when SRO is included.

due to the high $\mathrm{Fe}$ concentration. If one compares Fig. 10 with Figs. 1 and 8 one obtains a similar behavior of the minority-/majority-spin subsystem. The minority part of the DOS shows a hybridization among all cluster configurations whereas the majority part shows a separation in energy between the $\mathrm{FeFe} / \mathrm{FeCr}$ and $\mathrm{CrCr} / \mathrm{CrFe}$ clusters.

Figure 11 shows our results for the residual resistivity within the NL-CPA. In this plot we show again the three curves from Fig. 2 and additional some curves for different ordering situations. The NL-CPA results for disordered $\mathrm{Fe}_{1-x} \mathrm{Cr}_{x}$ shows satisfying agreement with the CPA results.

Experimentally it is observed that $\mathrm{Fe}_{1-x} \mathrm{Cr}_{x}$ tends for $x$ $<0.1$ to SRO and for $x>0.1$ to clustering ${ }^{9}$ whereas $\mathrm{Fe}_{1-x} \mathrm{~V}_{x}$ tends to SRO. ${ }^{38}$ Therefore we show in Fig. 11 NL-CPA calculations which simulate $\mathrm{SRO}\left(\mathrm{Fe}_{1-x} \mathrm{Cr}_{x}\right.$ and $\left.\mathrm{Fe}_{1-x} \mathrm{~V}_{x}\right)$ as well as clustering $\left(\mathrm{Fe}_{1-x} \mathrm{Cr}_{x}\right)$.

The important observation from our calculations is that the influence of ordering effects (SRO and clustering) on the residual resistivity is very small for these systems. In Ref. 15 the same formalism (using the same cluster sizes) was applied to bcc $\mathrm{Cu}_{1-x} \mathrm{Zn}_{x}$ as well as fcc $\mathrm{Ag}_{1-x} \mathrm{Pd}_{x}$ and gave a strong variation in the resistivity as a function of the ordering state. The small influence of ordering effects on the resistivity for $\mathrm{Fe}_{1-x} \mathrm{Cr}_{x}$ was also found experimentally by Mirebeau et $a l .{ }^{9}$ This shows that the formalism from Ref. 15 can handle various different ordering dependences of the residual resistivity. Nevertheless, one has to mention that we used in our calculations only small cluster sizes with two atoms. Therefore, it is not possible to preclude that bigger cluster sizes would have a bigger impact on our transport calculations.

\section{DISCUSSION AND SUMMARY}

The present work deals on an ab initio level with the anomalous concentration dependence of the residual resistiv- ity in $\mathrm{Fe}_{1-x} \mathrm{Cr}_{x}$ and $\mathrm{Fe}_{1-x} \mathrm{~V}_{x}$ alloys $(x \leq 0.2)$. Within CPA we obtain a Nordheim-type behavior for small $\mathrm{Cr} / \mathrm{V}$ concentrations (smaller $\approx 8 \% \mathrm{Cr}$, smaller $\approx 6 \% \mathrm{~V}$ ) and an approximately constant residual resistivity of $8 \mu \Omega \mathrm{cm}$ in the regime from $10 \%$ to $20 \% \mathrm{Cr}$ and $2 \mu \Omega \mathrm{cm}$ in the regime from $6 \%$ to $20 \% \mathrm{~V}$, respectively. Such a concentration dependence of the residual resistivity can be explained by the different contributions of the majority-/minority-spin channel to the conductivity. The BSFs of these spin channels are affected differently by the increase in the $\mathrm{Cr} / \mathrm{V}$ concentration as shown in Figs. 3 and 5. The broadening of the majority channel leads to a lifetime of these states approaching zero. Therefore, the contribution of the majority channel to the conductivity drops down, whereas the BSF for the minority channel is nearly unaffected by the increase in $\mathrm{Cr} / \mathrm{V}$ concentration. Hence, the contribution of these states to the conductivity is constant and responsible for the plateau in the regime from $10 \%$ to $20 \% \mathrm{Cr}(6 \%$ to $20 \% \mathrm{~V})$. These observations explain the anomalous resistivity behavior of Fe-rich antiferromagnetic alloys with comparable electronic structure properties as $\mathrm{Fe}_{1-x} \mathrm{Cr}_{x}$ and $\mathrm{Fe}_{1-x} \mathrm{~V}_{x}$, respectively. It turns out that the concentration independence of the residual resistivity is by no means anomalous for these materials. Therefore, we predict a similar behavior for other alloy systems from the same branch of the Slater-Pauling plot.

In addition, we investigated the influence of short-ranged correlations in the lattice site occupation by employing the NL-CPA formulation of the Kubo-Greenwood equation. The inclusion of such short-range effects has only little influence on the results.

The comparison of the $\mathrm{Fe}_{1-x} \mathrm{Cr}_{x}$ calculations with experiment shows satisfying agreement. The difference in the heights of the plateau, as compared to the experiment, could be attributed to impurities, lattice defects, grain boundaries, etc., which are always present in samples and therefore contained in the experimental data. Such imperfections, which have been neglected in the present calculations, lead in general to an increase in the measured resistivity. ${ }^{39}$

Our calculations show the initial linear increase albeit with a lower slope than seen in experiment. In Ref. 8 it is argued that this is a consequence of the limitations of the CPA for alloys with a dominant concentration of one constituent. However, it should be pointed out that our NL-CPA results confirm the single-site CPA data.

Our calculations reveal a plateau of the residual resistivity starting at the same $\mathrm{Cr}$ concentration as seen in the experiment (at $10 \% \mathrm{Cr}$ ). This is in variance to an earlier theoretical study ${ }^{8}$ which finds the starting point of the plateau only at $\approx 20 \% \mathrm{Cr}$.

\section{ACKNOWLEDGMENTS}

S.L. would like to thank the SFB 689 "Spinphänomene in reduzierten Dimensionen" for financial support. D.K. acknowledges support from the priority program of the DFG SPP 1145 "Modern and universal first-principles methods for many-electron systems in chemistry and physics." 
${ }^{1}$ R. M. Bozorth, Ferromagnetism (Van Nostrand, New York, 1951).

${ }^{2}$ N. F. Mott, Adv. Phys. 13, 325 (1964).

${ }^{3}$ J. Kübler, Theory of Itinerant Electron Magnetism (Clarendon, Oxford, 2000).

${ }^{4}$ J. Staunton, Rep. Prog. Phys. 57, 1289 (1994).

${ }^{5}$ A. P. Malozemoff, A. R. Williams, and V. L. Moruzzi, Phys. Rev. B 29, 1620 (1984).

${ }^{6}$ D. D. Johnson, F. J. Pinski, and J. B. Staunton, J. Appl. Phys. 61, 3715 (1987).

${ }^{7}$ A. Nikolaev, J. Phys.: Condens. Matter 11, 8633 (1999).

${ }^{8}$ Y. Y. Tsiovkin, A. N. Voloshinskii, V. V. Gapontsev, V. V. Ustinov, A. G. Obykhov, A. L. Nikolaev, I. A. Nekrasov, and A. V. Lukoyanov, Phys. Rev. B 72, 224204 (2005).

${ }^{9}$ I. Mirebeau, M. Hennion, and G. Parette, Phys. Rev. Lett. 53, 687 (1984).

${ }^{10}$ J. Cieślak, S. Dubiel, and B. Sepiol, J. Phys.: Condens. Matter 12, 6709 (2000).

${ }^{11}$ P. Olsson, I. A. Abrikosov, and J. Wallenius, Phys. Rev. B 73, 104416 (2006).

${ }^{12}$ R. Kubo, J. Phys. Soc. Jpn. 12, 570 (1957).

${ }^{13}$ D. A. Greenwood, Proc. Phys. Soc. London 71, 585 (1958).

${ }^{14}$ W. H. Butler, Phys. Rev. B 31, 3260 (1985).

${ }^{15}$ P. R. Tulip, J. B. Staunton, S. Lowitzer, D. Ködderitzsch, and H. Ebert, Phys. Rev. B 77, 165116 (2008).

${ }^{16}$ G. M. Stocks and W. H. Butler, Phys. Rev. Lett. 48, 55 (1982).

${ }^{17}$ J. S. Faulkner and G. M. Stocks, Phys. Rev. B 21, 3222 (1980).

${ }^{18}$ M. E. Rose, Relativistic Electron Theory (Wiley, New York, 1961).

${ }^{19}$ P. Soven, Phys. Rev. 156, 809 (1967).

${ }^{20}$ J. Banhart and H. Ebert, Europhys. Lett. 32, 517 (1995).

${ }^{21}$ J. Banhart and H. Ebert, Solid State Commun. 94, 445 (1995).

${ }^{22}$ H. Ebert, A. Vernes, and J. Banhart, Phys. Rev. B 54, 8479
(1996).

${ }^{23}$ J. Banhart, Phys. Rev. B 53, 7128 (1996).

${ }^{24}$ L. Dulca, J. Banhart, and G. Czycholl, Phys. Rev. B 61, 16502 (2000).

${ }^{25}$ A. Vernes, H. Ebert, and J. Banhart, Phys. Rev. B 68, 134404 (2003).

${ }^{26}$ M. Jarrell and H. R. Krishnamurthy, Phys. Rev. B 63, 125102 (2001).

${ }^{27}$ D. A. Rowlands, J. B. Staunton, and B. L. Györffy, Phys. Rev. B 67, 115109 (2003).

${ }^{28}$ D. A. Biava, S. Ghosh, D. D. Johnson, W. A. Shelton, and A. V. Smirnov, Phys. Rev. B 72, 113105 (2005).

${ }^{29}$ D. A. Rowlands, A. Ernst, B. L. Györffy, and J. B. Staunton, Phys. Rev. B 73, 165122 (2006).

${ }^{30}$ D. A. Rowlands, J. Phys.: Condens. Matter 18, 3179 (2006).

${ }^{31}$ E. N. Economou, Green's Functions in Quantum Physics (Springer-Verlag, New York, 2006).

${ }^{32}$ J. S. Faulkner, Prog. Mater. Sci. 27, 1 (1982).

${ }^{33}$ S. H. Vosko, L. Wilk, and M. Nusair, Can. J. Phys. 58, 1200 (1980).

${ }^{34}$ H. Ebert, in Electronic Structure and Physical Properties of Solids, edited by H. Dreyssé, Lecture Notes in Physics, Vol. 535, p. 191 (Springer, Berlin, 2000).

${ }^{35}$ H. Ebert, A. Vernes, and J. Banhart, Solid State Commun. 104, 243 (1997).

${ }^{36}$ S. U. Jen and S. A. Chang, Phys. Rev. B 47, 5822 (1993).

${ }^{37}$ V. Popescu, H. Ebert, N. Papanikolaou, R. Zeller, and P. H. Dederichs, J. Phys.: Condens. Matter 16, S5579 (2004).

${ }^{38}$ P. E. A. Turchi, L. Reinhard, and G. M. Stocks, Phys. Rev. B 50, 15542 (1994).

${ }^{39}$ P. L. Rossiter, The Electrical Resistivity of Metals and Alloys (Cambridge University Press, Cambridge, England, 1987). 